\title{
Evaluación del Compromiso Cardiovascular en niños en diálisis mediante el Índice de Masa Ventricular Izquierdo
}

\section{Left Ventricular Mass Index and Cardiovascular Compromise in children on dialysis}

\author{
Carolina Sugg H. ${ }^{\mathrm{a}, \mathrm{b}}$, Francisco Cano Sch. ${ }^{\mathrm{b}}$ \\ aPrograma Formación Nefrología Pediátrica

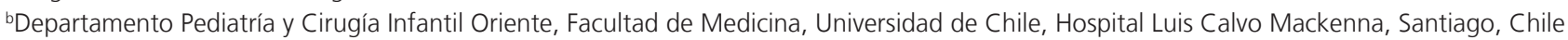

Recibido: 20 de febrero de 2020; Acepado: 30 de agosto de 2020

¿Qué se sabe del tema que trata este estudio?

La principal causa de muerte en la enfermedad renal crónica es la patología cardiovascular secundaria a los trastornos metabólicos, hidroelectroliticos, hipertensión arterial y sobrecarga de volumen propios de esta patología, en especial en etapas terminales.
¿Qué aporta este estudio a lo ya conocido?

Se destaca la importancia del diagnóstico y seguimiento del compromiso cardiovascular en niños en diálisis, resaltando la necesidad de un correcto diagnóstico de la hipertrofia ventricular izquierda en aquellos pacientes con un retraso de talla, característico de esta población.

\begin{abstract}
Resumen
Existe una estrecha relación entre enfermedad renal crónica (ERC) y enfermedad cardiovascular. Una de sus manifestaciones clínicas es la hipertrofia de ventrículo izquierdo (HVI), expresada como Indice de Masa Ventricular Izquierda (IMVI, gr $/ \mathrm{m}^{2.7}$ ). En pacientes portadores de ERC con retraso de crecimiento, el cálculo de IMVI debería ajustarse corrigiendo la edad para la talla. Objetivo: Comparar el IMVI corregido por edad para la talla, con el valor calculado por edad cronológica en niños con ERC en diálisis. Pacientes y Método: Estudio de corte transversal. Se analizan ecocardiografías de pacientes portadores de ERC en diálisis entre 1-18 años, enero de 2016 a julio 2017. Se evalua IMVI ajustando el valor expresado a $\mathrm{gr} / \mathrm{m}^{2.7}$ al percentil para la edad cronológica del niño, y luego se ajusta el valor a la edad corregida por la talla. Se usa estadística descriptiva y estudio de concordancia para las evaluaciones de IMVI calculado por edad cronológica y para edad corregida por talla. Resultados: Se incluyeron 26 pacientes, 75 ecocardiogramas. Un 56\% presentó HVI usando IMVI calculado por edad cronológica vs un $46,6 \%$ al corregir la edad para la talla. Al comparar los grupos de percentiles de IMVI-edad cronológica vs IMVI ajustado a la edad para la talla real, se observó que el 18,6\% de la muestra cambia de grupo de percentil, el $100 \%$ de ellos a un grupo de percentil inferior. La concordancia evaluada en base a coeficiente Kappa fue de 0,72 (concordancia perfecta $>0,8$ ), confirmando diferencias al ajustar el IMVI para la edad corregida por la talla. Conclusión: El cálculo de IMVI por edad cronológica sobreestima el compromiso cardiovascular en niños con ERC que característicamente tienen un retraso de talla. Los resultados sugieren que el cálculo de IMVI ajustado a la edad corregida por talla otorga mayor precisión al diagnóstico de hipertrofia ventricular izquierda en este grupo de pacientes.
\end{abstract}

Palabras clave:

Enfermedad Renal

Crónica;

Índice de Masa

Ventricular Izquierda;

Diálisis;

Retraso de Crecimiento

Correspondencia:

Carolina Sugg $H$.

carolasugg@yahoo.com 


\section{Abstract}

There is a close relationship between chronic kidney disease (CKD) and cardiovascular disease. One of its clinical manifestations is left ventricular hypertrophy (LVH), expressed as Left Ventricular Mass Index (LVMI gr/m $/ \mathrm{m}^{2.7}$ ). In CKD patients with growth retardation, the LVMI calculation should be adjusted by correcting age for length/height. Objective: To compare the age-corrected LVMI for length/height with the value calculated by chronological age in CKD children on dialysis. Patients and Method: Cross-sectional study. We analyzed echocardiographies of CKD children on dialysis aged between 1 and 18, from January 2016 to July 2017. LVMI was evaluated by adjusting the value expressed in $\mathrm{gr} / \mathrm{m}^{2.7}$ to the percentile for the chronological child's age, and then the value was adjusted to the age-corrected length/height. We used descriptive statistics and concordance study for LVMI assessments calculating by chronological age and for age-corrected length/height. Results: 26 patients were included and 75 echocardiograms. 56\% had left ventricular hypertrophy using chronological age versus $46.6 \%$ age-corrected LVMI for length/height. When comparing the percentile groups of LVMI-chronological age vs. age-adjusted LVMI for actual length/height, it was observed that $18.6 \%$ of the sample changed percentile groups, $100 \%$ of them to a lower percentile group. The agreement evaluated based on the Kappa coefficient was 0.72 (perfect agreement $>0.8$ ), confirming differences when adjusting the LVMI for age-corrected length/height. Conclusion: Calculating LVMI by chronological age overestimates the cardiovascular involvement in children with CKD who are characteristically stunted. The results suggest that the age-adjusted, length/height-corrected calculation of LVMI gives greater accuracy to the diagnosis of left ventricular hypertrophy in this group of patients.

\section{Keywords:}

Chronic Kidney

Disease;

Left Ventricular Mass

Index;

Dialysis;

Growth Retardation

\section{Introducción}

La enfermedad renal crónica (ERC) tiene una estrecha relación con la enfermedad cardiovascular siendo esta su mayor causa de mortalidad. Un 40 a $70 \%$ de los adultos que ingresan a un programa de diálisis ya tienen manifestaciones cardiovasculares y la mortalidad general por esta causa alcanza un $40 \%^{1}$. En niños con enfermedad renal crónica terminal (ERCT) la mortalidad secundaria a enfermedad cardiovascular oscila entre un 21 a $25 \%{ }^{2,3}$. Las manifestaciones clínicas de la enfermedad cardiovascular son secundarias a ateroesclerosis acelerada y cardiomiopatía. Esta última se puede presentar como hipertrofia de ventrículo izquierdo (HVI) concéntrica en situaciones de aumento de la postcarga, como dilatación de ventrículo izquierdo secundaria a sobrecarga de volumen o como disfunción sistólica por disminución de la contractibilidad ${ }^{4,5}$. A la fecha hay distintas herramientas diagnósticas para evaluar la presencia de cardiomiopatía como electrocardiograma, ecocardiografía y resonancia nuclear magnética, siendo la más utilizada por accesibilidad, rendimiento y costos el ecocardiograma ${ }^{6}$. En una cohorte canadiense con 432 individuos con ERCT que iniciaban diálisis se observó en un 45\% HVI concéntrica, en $28 \%$ dilatación de ventrículo izquierdo (VI) y un $16 \%$ de disfunción sistólica ${ }^{7}$. Según el registro del International Pediatric Peritonael Dialysis Network (IPPN) de 471 pacientes pediátricos en diálisis peritoneal evaluados con ecocardiograma un $30 \%$ presentaban HVI concéntrica, 18\% HVI excéntrica, 26\% remodelación concéntrica de VI y un $26 \%$ fueron informados como normales ${ }^{8}$.

Para calcular la masa de ventrículo izquierdo (MVI) por medio de la ecocardiografía se puede utilizar modo M ciego ó guíado por $2 \mathrm{D}$ o 3D. Todas las mediciones se realizan al final de la diástole. Quienes utilizan el modo M ó mediciones lineales en ecografía 2D del diámetro diastólico del VI y del espesor de la paredes se basan en fórmulas geométricas para calcular el volumen del miocárdio en VI, mientras que la ecografía 3D lo puede medir directamente ${ }^{9}$. La masa ventricular izquierda guarda directa relación con la masa magra corporal, parámetro difícil de estimar en la práctica clínica, por lo cual se ha usado la superficie corporal (SC) como aproximación, sin embargo la SC depende del peso y la talla, lo cual sugiere que individuos con sobrepeso u obesidad presentaran valores sesgados de MVI. Para corregir dicho sesgo, se ha definido la talla como mejor equivalente clínico a la masa magra. Los valores de MVI varían asi en función del genero, edad, talla y composicion corporal ${ }^{10}$, y su evaluación mejo$\mathrm{ra}$ al indexar la masa de ventrículo izquierdo (IMVI) a la talla elevada a la potencia 2,7 que confiere una mejor correlación con la masa magra. Esta última es la fórmula recomendada por la Academia Américana de Pediatría para diagnósticar HVI ortorgando como punto de corte en adultos valores $>51 \mathrm{~g} / \mathrm{m}^{2,7}$ y en niños $>38 \mathrm{~g} / \mathrm{m}^{2,7}{ }^{11-13}$. Este IMVI según Foster ${ }^{14}$ sería impreciso para el diagnóstico de HVI en niños debido a que las proporciones corporales cambian drásticamente durante la niñez y la relación de la MVI y la talla difiere 
a las distintas edades del desarrollo encontrando que la MVI ajustada a percentiles para la talla es superior. Khoury ${ }^{15}$ logra determinar percentiles de MVI e IMVI realizando ecocardiografías a 2273 niños sanos de 0 a 18 años y define HVI con valores mayores a percentil 95. A partir de este estudio se considera HVI en niños mayores de 9 años un IMVI $>45 \mathrm{~g} / \mathrm{m}^{2,7}$ y para niñas mayores de 9 años un IMVI $>40 \mathrm{~g} / \mathrm{m}^{2,7}$, pero para niños menores este valor varia ampliamente con la edad, por lo que no recomienda utilizarlo ${ }^{15}$.

De los niños con ERCT un 68\% presenta talla baja $(<1,88$ DS $)$ y a pesar del avance clínico y del manejo terapeútico, un 35 a $50 \%$ permanece con compromiso de talla en la vida adulta ${ }^{16,17}$. Es por este motivo que planteamos que en niños con ERCT podría ser más preciso utilizar los percentiles descritos por Khoury para evaluar el IMVI corrigiendo la edad por la talla del niño.

El objetivo de este estudio es determinar si el cálculo del índice de masa de ventrículo izquierdo corregido por edad para la talla difiere del valor calculado por edad cronológica en niños portadores de ERC en diálisis crónica.

\section{Pacientes y Método}

Estudio observacional analítico transversal retrospectivo en pacientes pediátricos portadores de ERCT activos en programa de diálisis peritoneal y hemodiálisis crónica del Hospital Luis Calvo Mackenna durante enero del 2016 a julio del 2017, rango de edad entre 1 y 18 años. Criterios de inclusión son niños con ERCT en peritoneodiálisis o hemodiálisis entre 1 y 18 años. Se excluyen del estudio niños con cardiopatía congénita y cromosomopatías.

Se recolectaron desde su ingreso a programa de diálisis datos desde fichas clínicas para variables demográficas: género, edad cronológica; variables clínicas: peso $(\mathrm{kg})$; estatura $(\mathrm{cm})$; presión arterial sistólica (PS) y diastólica (PD) en $\mathrm{mmHg}$ clasificando valores como mayor o menor a percentil 95 de acuerdo a edad, género y talla según tablas de presión arterial publicadas por Academia Américana de Pediatría el año 2017 (13); etiología de ERC (anomalías estructurales congénitas del tracto urinario, glomerulopatías, alteraciones hereditarias, nefropatía vascular, otras y de etiología no precisada); tiempo de ERCT (meses); tiempo de terapia de reemplazo renal (TRR), diálisis peritoneal (DP) y/o hemodiálisis (HD). Parámetros de laboratorio: hematocrito $(\%)$, hemoglobina $(\mathrm{gr} / \mathrm{dl})$, ferritina plasmática $(\mathrm{ng} / \mathrm{ml})$, albúmina $(\mathrm{gr} / \mathrm{dl})$, creatinina plasmática $(\mathrm{mg} / \mathrm{dl})$ y clearence de creatinina estimado por Schwartz $(\mathrm{ml} / \mathrm{min})\left[0,413{ }^{\star}\right.$ talla $(\mathrm{cm}) /$ creatinina plasmática $(\mathrm{mg} / \mathrm{dl})]^{18}$, función renal residual $(\mathrm{FRR})$ considerando diuresis $>100 \mathrm{ml} /$ día, calcemia $(\mathrm{mg} / \mathrm{dl})$, fosfemia ( $\mathrm{mg} / \mathrm{dl})$, PTH intacta $(\mathrm{pg} / \mathrm{ml})$ y niveles de 25 $\mathrm{OH}$ vitamina $\mathrm{D}(\mathrm{ng} / \mathrm{ml})$ En aquellos niños(as) en $\mathrm{HD}$ se obtuvo Kt/V y para los niños(as) en DP Kt/V peritoneal del mes de realizada la ecocardiografía.

\section{Crecimiento}

Para talla se utilizó patrón de referencia OMS para niños y niñas menores de 5 años y tablas NCHS para mayores de 5 años. Se convirtió la estatura en puntaje $\mathrm{Z}$ (puntaje de la deviación estandar) de acuerdo a las tablas de talla para la edad de la OMS y NCHS para poder expresar en forma estadística los datos ${ }^{19-21}$.

Se definió edad corregida para la talla como la edad en que la talla del niño corresponde a su percentil 50 para género según OMS en menores de 5 años y NCHS para mayores de 5 años.

\section{Cardiovascular}

Se analizaron las ecocardiografías realizadas en Hospital Luis Calvo Mackenna por 2 cardiólogos pediátricos con equipo Vivid E9 GE Healthcare ${ }^{\circledR}$. Se incluyeron ecocardiografías realizadas con intervalos de al menos 6 meses en el grupo en estudio. Se obtuvieron datos apartir de las ecocardiografías realizadas por medio de Modo M guíado por 2D de diámetro de pared posterior de VI (DPPVI), diámetro de septum intreventricular (DSIV) y diamétro de ventrículo izquierdo al fín del diástole (DVIFD) en centímetros, y se cálculo la MVI utilizando el programa http://lvmass.parameterz.com basado en fórmulas recomendadas por la American Society of Echocardiography ${ }^{22}$. Se estimó el IMVI según MVI/talla ${ }^{2,7}$. Se adjudicó el rango de percentil de MVI e IMVI según curvas y tablas de Khoury et al. por edad y luego por edad corregida por la ta$1 \mathrm{a}^{15}$. Dado que esta tabla no presenta el MVI e IMVI en forma de variable continua, sino que muestra valores de corte en percentiles 10, 25, 50, 75, 90 y 95, se usó el rango interpercentiles en el cual se situa cada caso. De esta forma los pacientes se agruparon en rangos de percentiles: grupo $1(<\mathrm{p} 10)$, grupo 2 (p 10-25), grupo 3 (p 25-50), grupo 4 (p 50-75), grupo 5 (p 75-90), grupo 6 (p 90-95) y grupo 7 (> p95). Se definió como HVI aquellos valores superiores al percentil 95 de MVI e IMVI según Khoury et al. ${ }^{15}$.

Este trabajo fué evaluado por el Comité de Ética e Investigación de Seres Humanos de la Facultad de Medicina de la Universidad de Chile. Se solicitó consentimiento informado a tutores legales y asentimiento informado para niños mayores de 12 años.

\section{Análisis estadístico}

Se utilizó para la tabulación de los datos planilla Excel, el análisis de los datos se realizó con dos colas. Para el análisis estadístico se utilizó STATA 11.0. Las varia- 
Tabla 1. Características generales de los pacientes al momento del ecocardiograma

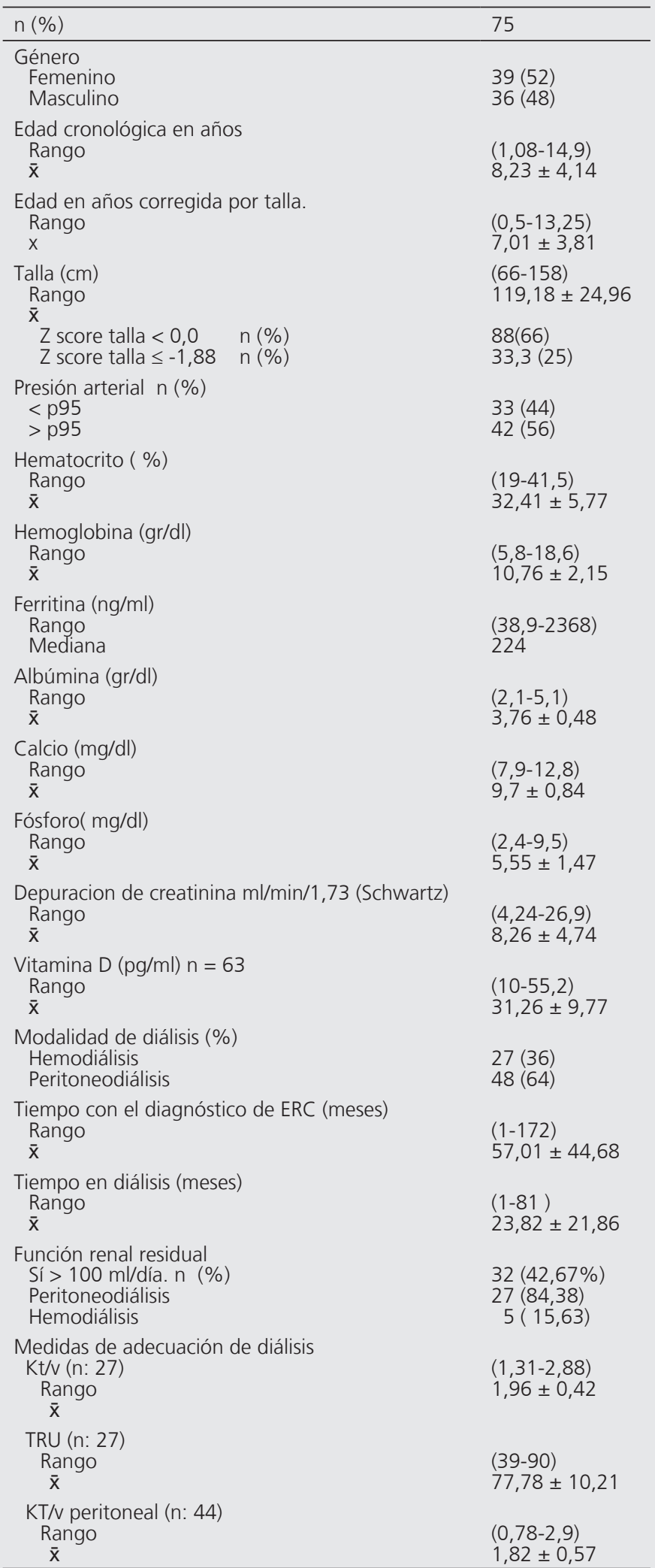

Datos son presentados como número (\%), promedio (x), \pm desviación estándar, rango y mediana. ERC: Enfermedad Renal Crónica. KtN: dosis de dialisis. TRU: Tasa de Reduccion de Urea. bles categóricas se expresaron como valor absoluto y porcentaje, las variables continuas con distribución normal como media aritmética \pm desviación estándar y para aquellas variables no paramétricas como mediana e intervalo intercuartiles (percentil25- percentil 75). Se utilizó estudio de concordancia con cálculo de coeficiente kappa y alfa de Cronbach para la evaluación de MVI e IMVI por edad cronológica versus edad corregida por la talla, se consideró concordancia para ambos coeficientes con un valor $>0,8^{23}$. Este estudio utilizó una muestra de oportunidad ya que se seleccionaron todas las ecocardiografías de sujetos en diálisis crónica y en el cuál no se puede medir un error de estimación. Se incluyeron estudios ecocardiográficos de un mismo paciente separados al menos por períodos de 6 meses, dado que la variable de interés correspondió al IMVI.

\section{Resultados}

Se seleccionaron inicialmente 30 pacientes portadores de ERCT activos en programa de diálisis peritoneal y hemodiálisis crónica del Hospital Luis Calvo Mackenna, enero 2016 a julio 2017, de los cuales se excluyeron 2 niños con diagnóstico de cardiopatía congénita y cromosomopatía y 2 por no poseer ecocardiograma de cardiólogo pediátrico del Hospital Luis Calvo Mackenna. Etiología de ERC, 53,9\% presentaba anomalías estructurales congénitas del tracto urinario, 26,9\% glomerulopatías, 3,9\% alteraciones hereditarias, $7,7 \%$ nefropatía vascular, $3,9 \%$ otras y $3,9 \%$ de etiología no precisada. El promedio de edad fue de 8,2 $\pm 4,1$ años. La edad promedio corregida por la talla fue de $7,01 \pm 3,8$ años. Un $33 \%$ de la muestra presentaba talla baja (<1,88 DS). Las características demográficas y de laboratorio se presentan en la tabla 1.

Durante el periódo de análisis del grupo se obtuvieron 75 ecocardiogramas, en promedio 2,88 ecocardiogramas por paciente (rango: 1-10), con un intervalo de tiempo entre ecocardiografías en promedio de 30,7 meses (7-143 meses). Al evaluar la MVI y el IMVI por edad cronológica un $37,3 \%$ y un $56 \%$ de los estudios correspondió a una MVI y un IMVI > p95, respectivamente. Al ajustar por edad corregida por la talla, se observó un $50,7 \%$ con MVI > p95 y un 46,7\% con IMVI $>$ p95 (tablas 2 y 3 ).

Para evaluar la consistencia interna de los instrumentos de medida utilizados, con y sin ajuste por la edad corregida para la talla, se utilizó coeficiente de correlación alfa de Cronbach. Se obtuvo un coeficiente de 0,92 para MVI y de 0,97 para IMVI, posteriormente se analizó la concordancia entre ambos instrumentos de medición descontando el acuerdo obtenido por el azar, utilizando coeficiente de correlación kappa de Cohen, resultando un coeficiente de 0,42 para las medidas relacionadas a MVI y de 0,72 para las de IMVI. 
Tabla 2. Grupos de rangos de percentiles MVI e IMVI por edad cronológica

\begin{tabular}{ccc}
\hline Grupo (Rango percentiles*) & MVI $(n, \%) n: 75$ & IMVI $(n, \%) n: 75$ \\
\hline Grupo 1 (<p 10) & $10(13,33)$ & $4(5,33)$ \\
Grupo 2 (p 10-25) & $4(5,33)$ & $3(4)$ \\
Grupo 3 (p 25-50) & $13(17,33)$ & $8(10,67)$ \\
Grupo 4 (p 50-75) & $8(10,67)$ & $4(5,33)$ \\
Gupo 5 (p 75-90) & $5(6,67)$ & $10(13,33)$ \\
Grupo 6 (p 90-95) & $7(9,33)$ & $4(5,33)$ \\
Grupo 7 (> p 95) & $28(37,33)$ & $42(56)$ \\
\hline
\end{tabular}

*Rango de percentiles a partir de tablas de Khoury et al. ${ }^{15}$. MVI: Masa Ventricular Izquierda. IMVI: Indice de MVI.

Tabla 3. Grupo de rango de percentiles MVI e IMVI para la edad corregida por la talla

\begin{tabular}{ccc}
\hline Grupo (Rango Percentiles*) & MVI ajustado $(\mathrm{n}, \%)$ & IMVI ajustado $(\mathrm{n}, \%)$ \\
\hline Grupo 1 $(<\mathrm{p} \mathrm{10})$ & $6(8)$ & $5(6,67)$ \\
Grupo 2 $(\mathrm{p} \mathrm{10-25)}$ & $1(1,33)$ & $6(8)$ \\
Grupo 3 (p 25-50) & $7(9,33)$ & $5(6,67)$ \\
Grupo 4 (p 50-75) & $10(13,33)$ & $6(8)$ \\
Gupo 5 (p 75-90) & $9(12)$ & $12(16)$ \\
Grupo 6 (p 90-95) & $4(5,33)$ & $6(8)$ \\
Grupo 7 (> p 95) & $38(50,67)$ & $35(46,67)$ \\
\hline
\end{tabular}

*Rango de percentiles a partir de tablas de Khoury et al. ${ }^{15}$. MVI: Masa Ventricular Izquierda. IMVI: Indice de MVI.

Al comparar los grupos de percentiles de IMVI con IMVI ajustado a la edad para la talla se observó que el $18,6 \%$ de la muestra cambió de grupo de percentil, el $100 \%$ de ellos a un grupo de percentil inferior. De los casos que cambiaron de grupo, un 50\% dejó de pertenecer al grupo 7 que confiere el diagnóstico de HVI.

\section{Discusión}

La hipertrofia ventricular izquierda es una de las complicaciones que debe ser evaluada en forma periódica en los pacientes con ERCT, siendo conocido que se asocia a un peor pronóstico cardiovascular. El método ampliamente utilizado en la práctica clínica para diagnosticar esta complicación es el ecocardiograma, tanto por su accesibilidad como por su costo. En la práctica clínica de nefrología pediátrica es habitual calcular el IMVI en gramos/m (talla) elevado a la potencia $2,7^{10,11,13}$, lo cual permite fijar un valor de corte por sobre el cual se diagnóstica una hipertrofia ventricular. Se usa la talla como un mejor parámetro clínico relacionado a la masa magra corporal, sin embargo, dado que la MVI varía en función de los cambios de la masa magra/talla según la edad, siendo mayor a menor edad, Foster et al. ${ }^{14}$ han sugerido que un valor único de corte no es aplicable en pediatría, en especial en menores de 9 años, por lo que han demostrado que el valor de IMVI gr/talla ${ }^{2.7}$, debe ser ajustado usando los percentiles de talla de la población estudiada, para ajustar a la talla/edad cronológica y así representar efectivamente el percentil 95 en cada paciente. Por ello, autores como Khoury et al..$^{15}$ han propuesto tablas que corrigen la MVI y el IMVI de acuerdo a edad y género, pero ajustando al percentil de talla/edad cronológica. Ello nos permite plantearnos que un valor " $x$ " de talla en un paciente de una edad de 7 años cronológica, sea leído en función de la edad corregida para la talla real del paciente enfermo renal crónico, que podría corresponder a un niño de "5" años.

En nuestro estudio encontramos que un 56\% de los casos cumple con el criterio de HVI similar a los descrito por Lehmman ${ }^{24}$, pero cuando se ajusta por la edad corregida por la talla la HVI disminuye a un $46,6 \%$, al analizar el grado de concordancia de este resultado mediante escala de valoración kappa nos encontramos que el coeficiente tanto para MVI e IMVI inferior a nuestro punto de cohorte de 0,8 , siendo posible concluir que sí existen diferencias en los rangos de percentiles de MVI e IMVI obtenidos al aplicar la corrección por edad corregida para la talla.

Este estudio contempló el análisis de 75 ecocardiogramas en 26 pacientes, lo cual representa un promedio de 2,9 ecocardiogramas por cada paciente con un 
intervalo mínimo entre cada examen de 6 meses. Este punto podría ser considerado una potencial fuente de sesgo si las variables analizadas fuesen estáticas en el tiempo, sin embargo el análisis en detalle de cada caso permite observar un hecho característico de los pacientes pediátricos en diálisis, cual es que un mismo sujeto presenta cambios significativos en su crecimiento (T/E), al igual que en el estado cardiovascular, en particular en edades pre adolescencia, lo que permite utilizar este examen en un mismo paciente en distintos períodos de tiempo para obtener la información que el objetivo persigue. Al analizar en detalle cómo se mueven estos grupos de rangos de percentiles al corregir la edad por la talla del niño(a) observamos que un $100 \%$ de los casos que se movilizan de grupo lo hacían a un grupo de percentil inferior lo que refleja un sobre diagnóstico de HVI en $10 \%$ de los ecocardiogramas y a un $30 \%$ de los sujetos incluidos en este estudio. En pacientes pediátricos en diálisis, la sobrecarga de volumen es frecuente y una de las principales causas de hipertensión e hipertrofia ventricular, lo cual hace especialmente importante un diagnóstico exacto del IMVI como parámetro objetivo de este compromiso cardiovascular. El sobre diagnóstico de HVI puede significar efectuar ajustes innecesarios en la terapia, basándose en una hipertrofia ventricular sobrestimada al no ajustar el IMVI de acuerdo a la talla/edad corregida del paciente. Una excesiva ultrafiltración (UF) tanto en PD como HD puede llevar a efectos no deseados como la hipotensión. Se ha visto en pacientes pediátricos que la hipotensión intradiálisis en HD convencional produce toxicidad miocárdica con disminución de la fracción de acortamiento del $\mathrm{VI}^{25}$. Por otro lado una excesiva UF nos lleva a una pérdida precoz de la función renal residual que nos dificulta mantener la euvolemia, creando la necesidad de usar soluciones hipertónicas en PD para optimizar la UF con su conocido efecto deletero sobre la membrana peritoneal, secundario a disminución de angiogénesis y fibrosis peritoneal sumando a ellos un peor control metabólico secundario a hiperglicemia producida por soluciones hipertóni$\operatorname{cas}^{26,27}$. La pérdida de la función renal residual impide la depuración de moléculas de mayor peso molecular, aumenta el estado inflamatorio y se asocia al menos en adultos a un mayor riesgo de mortalidad ${ }^{28-32}$.

Este estudio tiene las limitaciones propias de un diseño retrospectivo, si bien el ecocardiograma es muy útil para evaluar complicaciones cardiovasculares en este grupo de pacientes hubiese sido más enriquecedor estimar si la HVI se debe a sobrecarga de volumen y/o un aumento de la postcarga apoyándonos no sólo en el informe ecocardiográfico sino también con en el uso herramientas complementarias para evaluar la sobrecarga de volumen como son bioimepdanciometría e índice de colapsabilidad de la vena cava inferior y de- terminar la existencia hipertensión arterial con monitoreo de presión arterial ambulatorio ${ }^{33,34}$. Como todo examen de laboratorio, se debe tener en consideración un potencial sesgo derivado del informe ecocardiográfico realizado por un solo observador, sin análisis de concordancia intra o interobservadores, aspecto que en la práctica clínica diaria no es posible de realizar, por lo cual se usaron los informes de 2 expertos cardiólogos pediátricos del Centro hospitalario.

Frente a los resultados obtenidos en este estudio se sugiere considerar la talla de los pacientes al momento de la evaluación del IMVI, ya que las tablas confeccionados por Khoury et al. se construyeron a partir de una población de niños sanos que presentan una curva de distribución talla/edad normal, y la talla en niños con ERCT hace que en este grupo la distribución de la estatura corresponda a percentiles más bajos lo que los aleja de la posibilidad de encasillarlos en un grupo de distribución normal y favorece un sobre diagnóstico del compromiso cardiovascular, fenómeno observado en este estudio.

En conclusión, el compromiso cardiovascular en diálisis crónica debe ser diagnosticado con exactitud para poder adoptar medidas enfocadas en optimizar los tratamientos. En el grupo estudiado el tradicional cálculo de IMVI realizado por edad cronológica sobreestima el compromiso cardiovascular por lo cual se debe evaluar el ajuste de IMVI por edad corregida para la talla del paciente. Esta forma de evaluar la HVI puede ser importante en un grupo de pacientes que característicamente tiene un retraso de talla propio de la ERC.

\section{Responsabilidades Éticas}

Protección de personas y animales: Los autores declaran que los procedimientos seguidos se conformaron a las normas éticas del comité de experimentación humana responsable y de acuerdo con la Asociación Médica Mundial y la Declaración de Helsinki.

Confidencialidad de los datos: Los autores declaran que han seguido los protocolos de su centro de trabajo sobre la publicación de datos de pacientes.

Derecho a la privacidad y consentimiento informado: Los autores han obtenido el consentimiento informado de los pacientes y/o sujetos referidos en el artículo. Este documento obra en poder del autor de correspondencia.

\section{Conflicto de intereses}

Los autores declaran no tener conflicto de intereses. 


\section{Referencias}

1. Keith DS, Nichols GA, Gullion CM, Brown JB, Smith DH. Longitudinal follow-up and outcomes among a population with chronic kidney disease in a large managed care organization. Arch Intern Med. 2004;164:659-63.

2. Parekk R, Carroll C, Wolfe R, Port F. Cardiovascular mortality in children and young adults with end stage kidney disease. J Pediatr 2002;141:191-7.

3. North American pediatric renal trials and collaborative studies. NAPRTCS. 2011. https://web.emmes.com/study/ped/ annlrept/annlrept.html.

4. Mitsnefes M. Cardiovascular complications of pediatric chronic kidney disease. Pediatr Nephrol 2008;23:27-39.

5. Bardají A, Martínez- Vea A. Enfermedad renal crónica y corazón. Un continuo evolutivo. Rev Esp Cardiol. 2008;61(Supl 2):41-51.

6. Llancaqueo V. Hipertrofia ventricular izquierda como factor de riesgo cardiovascular en el paciente hipertenso. Rev. Med. Clin. Las Condes. 2012;23(6):707-14.

7. Parfrey PS, Foley RN, Harnett JD, Kent GM, Murray DC and Barre PE. Outcome and risk factors for left ventricular disorders in chronic uremia. Nephrol Dial Transplant. 1996;11:1277-85.

8. Borzych D, Greenbaum L, Patel, et al. Left ventricular hypertrophy in children receiving peritoneal dialysis: A Report from the IPPN Registry. www.pedpd.org.

9. Grattan M, Mertens M.

Echocardiographic assessment of ventricular function in pediatric patients: a comprehensive guide. Future Cardiology 2014;10:511-23.

10. Boryzch D, Sevcan B, Bakkaloglu A, et al. Defining left ventricular hypertrohy in children on peritoneal dialysis. Clin J Am Soc Nephrolol 2011;6:1934-43.

11. De Simone D, Daniels SR, Deveraux RB et al. Left ventricular mass and body size in normotensive children and adults; assessment of allometric relations and impact of overweight. J Am Coll Cardiol 1992;20:1251-60.
12. De Simone, Deveraux RB, Daniels SR, et al. Effect of growth on variability of left ventricular mass: assessment of allometric signals in adults and children and their capacity to predict cardiovascular risk. J am Coll Cardiol 1995;25:1056-62.

13. Clinical practice guideline for screening and management of high blood pressure in children and adolescents. Pediatrics 2017;140(3): e20171904.

14. Foster BJ, Mackie AS, Mitsnefes M et al. A novel method of expressing left ventricular mass relative to body size in children. Circulation 2008;117:2769-75.

15. Khoury P, Mitsnefes M, Daniels S et al. Age- especific reference intervals for indexed left ventricular mass in children. Am Soc Echocardiogr 2009;22:709-14.

16. Furth S, Hwang W, Yang $C$ et al. Growth failure, risk of hospitalization and death for children with end-stage renal disease. Pediatr Nephrol 2002;17:450-55.

17. Heffner D, Schaefer F, Nissel R et al. Efect of growth hormone treatment on the adult height of children with chronic renal failure. N Engl J Med 2000;343:923-30.

18. Schwartz G, Muñoz A. Schneider M, et al. New equations to estimate GFR in children with CKD. J Am Soc Nephrol 2009;20:629-37.

19. Pombo M, Castro- Feijóo, Cabanas Rodriguez P. El niño con talla baja. Protoc diagn ter pediatr. 2011;1:236-54.

20. http://www. cdc.gov/growthcharts.

21. http:// www.who.int/childgrowth/ standards/curvas_por_indicadores.

22. López L, Colan S, Frommelt, et al. Recommendations for quantification methods during the performance of a pediatric echocardiogram: a report from the pediatric measurements writingroup of the American Society of Echocardiography Pediatric and Congenital Heart Disease CouncilJ. J Am Soc Echocardiogr 2010;23:465-95.

23. Landis JR, Koch G.G. The measurement of observer agreement for categorical data. Biometrics. 1977;33:159-74.

24. Lehmann P, Cano F. Compromiso cardiovascular en pacientes pediátricos en diálisis peritoneal crónica. Rev Chil Pediatr. 2017;88(2):236-242.
25. Hothi DK, Ress L, Marek J et al. Pediatric myocardial stunning underscores the cardiac toxicity of conventional hemodyalisis treatments. Clin J Am Soc Nephrol 2009;4:790-7.

26. Ha IS, Yap HK, Munarriz Rl et al. Risk factors for loss of residual renal function in children treated with chronic peritonel dialysis. Kidney Int. 2015;88:605-13.

27. Kim CD, Kwon HM, Park SH et al. Effects of low glucose degradation products peritoneal dialysis fluid on the peritoneal fibrosis and vascularization in a chronic rat model. Ther Apher Dial 2007;11:5664.

28. Kim YL. Update on mechanisms of ultrafiltration failure. Perit Dial Int. 2009; Suppl 2:S123-7.

29. HU SL, Joshi P, Kaplan M, et al. Rapid change in residual renal function decline is associated with lower survival and worse residual renal function preservation in peritoneal dialysis patients. Perit Dial Int 2017;37:477-81.

30. Rocco MV et al. Risk factors for early mortality in U.S peritoneal dialysis patients: Impact of residual function. Perit Dial Int. 2002;22:371-9.

31. Canusa Churchill DN, Taylor DW, Keshaviah PR, and the CANUSA Peritoneal Dialysis Study Group. Adequacy of dialysis and nutrition in continuous peritoneal dialysis: association with clinical outcomes. J Am Soc Nephrol 1996;7:198-207.

32. Ademex Paniagua R, Amato D, Vonesh E et al; Mexican Nephrology Collaborative Study Group. Effects of increased peritoneal clearances on mortality rates in peritoneal dialysis: ADEMEX, a prospective, randomized, controlled trial. J Am Soc Nephrol 2002;13:1307-20.

33. Hayes W, Paglialonga F. Assessment and management of fluid overload in children on dialysis. Pediatr Nephrol. 2018 Mar 9. doi: 10.1007/s00467-0183916-4.

34. Paglialonga F, Consolo S, Edefonti A. Blood pressure management in children on dialysis. Pediatr Nephrol. Pediatr Nephrol. 2018;33:239-50. 